\title{
Molecular Analysis of Diversity Presents in Brassica juncea genotypes with the Help of SSR Markers
}

\author{
Nupur Saini ${ }^{* *}$ and Archana N. Rai ${ }^{2}$ \\ ${ }^{1}$ Department of Plant Molecular Biology and Biotechnology, IGKV, Raipur 492012, India \\ ${ }^{2}$ Nuclear Agriculture and Biotechnology Division, Bhabha Atomic Research Centre, \\ Mumbai 400085, India \\ *Corresponding author
}

\section{A B S T R A C T}

Brassica juncea is one of the most important oilseed crops of India, but its genetic diversity is still not explored properly. A better understanding on

Keywords

Brassica juncea, SSR markers, Polymorphism and genetic diversity

\section{Article Info}

Accepted:

22 June 2020

Available Online:

10 July 2020 this topic is a prerequisite for the better utilization of genotypes for breeding programs as well as in crop improvement. This study was undertaken to identify the polymorphism among thirty six genotypes including pure lines/varieties/ accession lines of different agro-climatic areas using fifteen SSR markers. All the thirty six genotypes were raised in pots for extraction of genomic DNA from seven days old seedlings. Amplification of the genomic DNA was carried out using a fifteen primer pairs. Out of the fifteen primers tested, seven reported polymorphism and a total of 32 alleles were amplified. The number of alleles per primer varied from one to three, with an average of 1.5 fragments, while the size of the fragments ranged from $200 \mathrm{bp}$ to $400 \mathrm{bp}$. Jaccard's similarity coefficients based on SSR data ranged from 0.36 to 0.1 . The study focuses on using SSR markers as a stronger and reliable tool for diversity studies.

\section{Introduction}

The family Brassicaceae, includes about 3,500 species and 350 genera and Brassica juncea is one of the most important crop of this family. Following soybean (Glycine max L.) and palm (Elaeis guineensis Jacq.), it is the third important oilseed crop in the worldwide. It is commonly grown in countries such as India, Canada, China, Pakistan, Poland, Bangladesh, Sweden and France. Brassica species occupies first position with 20.23 percent of total area under cultivation among all the oilseeds in India (USDA, 2014). Among different Brassica species four of them viz. B. napus, B. juncea, B. carinata 
and $B$. rapa commonly known as rapeseedmustard, are cultivated in about 6.39 million ha area and have yield of 7.41 million tons in India (Kumar, 2015). Out of these, Indian mustard contributes more than 80 percent to the total rapeseed-mustard production of the country.

But after yellow revolution, the production and productivity of mustard in India seems to be static from last one decade whereas it's productivity is hovering between 1 to $1.2 \mathrm{t} / \mathrm{ha}$, which is much lower than the world's average productivity of $1.98 \mathrm{t} / \mathrm{ha}$ (FAOSTAT, 2014). However the tremendously increasing population and improving life standards, demands for per capita oil have increased. To fulfill the current oil requirements, there is an urgent urge to increase the yield potential of $B$. juncea with the help of genetic interventions.

For maximizing the potential of any crop for its improvement, adaptation against different unfavorable environment and breeding depends mainly on the level of genetic diversity it holds. Knowledge on genetic diversity would further help the breeder and geneticist to understand the genetic makeup more clearly and help them to predict which combinations would produce the better offsprings (Hu et al., 2007). Different morphological, biochemical and molecular approaches can be used to determine genetic diversity present among individuals or populations (Mohammadi and Prasanna, 2003).

Out of all the different markers available for determining genetic diversity among plants, molecular markers are considered to be more precise, efficient and reliable (Mishra et al., 2011). Therefore, in the present study to determine the genetic diversity of thirty six $B$. juncea genotypes of different geographic origin SSR (Single sequence repeat) markers are used.

\section{Materials and Methods}

\section{Plant material}

Thirty six $B$. juncea genotypes, including purelines/varieties/ accession lines from different agro-climatic zones of India were taken up for this study (Appendix 1 and 2).

\section{Molecular marker analysis}

DNA from thirty six genotypes was isolated from young seedling (7 days old) using CTAB (Cetyl Trimethyl Ammonium Bromide) method (Doyle and Doyle, 1990). DNA so extracted was purified treating with phenol. After purification, DNA was quantified by using a Spectrophotometer at UV absorption of $260 \mathrm{~nm}$ assuming $1 \mathrm{OD}$ at $260 \mathrm{~nm}$ is equal to $50 \mu \mathrm{g}$ of DNA. The concentration of DNA was estimated from the following formula:

Concentration of DNA $(\mu \mathrm{g} / \mathrm{ml})=\mathrm{A} 260 \times 50 \mathrm{x}$ dilution factor.

Further, DNA samples were analyzed using $0.8 \%$ TAE- agarose gel to check its integrity (Fig 1). It was then diluted to $30 \mathrm{ng} / \mu \mathrm{L}$ for PCR analysis. Fifteen (15) SSR primer pairs were used to study DNA polymorphism by carrying out the DNA amplification in PCR (Appendix 3). The amplification reaction was carried out in $20 \mu \mathrm{L}$ reaction mixture containing $10 \mathrm{X}$ Taq buffer, $25 \mathrm{mM} \mathrm{MgCl}_{2}$, $10 \mathrm{mM}$ dNTPs, 10pmole primers, 1unit $/ \mu \mathrm{L}$ Taq DNA polymerase and 30ng template DNA. DNA amplification was programmed for 35 cycles in PCR with a program comprising of an initial denaturation cycle for five minutes at $95^{\circ} \mathrm{C}$. Each cycle consisted of a denaturation step at $95^{\circ} \mathrm{C}$ for thirty seconds, an annealing step at $55^{\circ} \mathrm{C}$ for thirty seconds, and an extension step at $72^{\circ} \mathrm{C}$ for one minutes, following by extension cycle for ten minutes at $72^{\circ} \mathrm{C}$ in the final cycle. The 
amplified fragments were then resolved on $2.5 \%$ agarose gel (Fig 2).

\section{Results and Discussion}

Bands were scored on the basis of presence and absence of bands i.e. one for presence and zero for absence of bands.

Out of the fifteen SSR markers, seven SSR were detected polymorphic with 32 amplified alleles. The number of alleles per primer varied from one to three, with an average of 1.5 fragments, while the size of the fragments ranged from $200 \mathrm{bp}$ to $400 \mathrm{bp}$. Jaccard's similarity coefficients based on SSR data ranged from 0.36 to 0 . 1 . Dendrogram based on the unweighted pair group method of arithmetic mean (UPGMA) was constructed to cluster genotypes into different groups using Jaccard's similarity coefficient (Fig 3).
The UPGMA based dendrogram representing genetic similarity among different accessions grouped the thirty six genotypes into two clusters which are then divided into 4 subgroups and many sub -sub groups. First cluter included only rohini variety from CSAUAT, Kanpur. The second cluster comprised of four sub groups. Subgroup I included CG local and IC- 405235, subgroup II had Bio 902, GM-2 and P. Jaikisan and subgroup III consists of Pusa Bahar, RGN-48 and JMM-927. Subgroup IV is further divided into two sub - subgroups. I sub - sub group comprised of JM-2, NPJ-124, RH-187, GM-3 and Mahak. 22 genotype fall in II sub subgroup which includes NPJ -113, RGN-73, RB - 50, IC - 264986, RCC-4, NRHBH -101, Geeta, RH - 189, Varuna, Mahiar, IC113037, P.Bold, RL-1359, TPM-1, Maya, NPJ-112, TM-4, IC-26513, Laxmi, RNGDR -02 and Kranti

\section{Appendix.1 List of Genotypes used in present study}

\begin{tabular}{|r|c|c|c|}
\hline S.No. & Genotypes Name & S.No. & Genotype Name \\
\hline $\mathbf{1 .}$ & P.BOLD & 17. & KRANTI \\
\hline $\mathbf{2 .}$ & P.JAIKISAN & 18. & JM-2 \\
\hline $\mathbf{3 .}$ & MAHAK & 19. & RL-1359 \\
\hline $\mathbf{4 .}$ & MAHIAR & 20. & GM-3 \\
\hline $\mathbf{5 .}$ & RJN-48 & 21. & GM-2 \\
\hline $\mathbf{6 .}$ & RJN-73 & 22. & RB-50 \\
\hline $\mathbf{7 .}$ & P.BAHAR & 23. & RNGDR-02 \\
\hline $\mathbf{8 .}$ & TPM-1 & 24. & RH-189 \\
\hline $\mathbf{9 .}$ & TM-1 & 25. & NPJ-112 \\
\hline $\mathbf{1 0 .}$ & TM-4 & 26. & NPJ-113 \\
\hline $\mathbf{1 1 .}$ & C.G LOCAL & 27. & NPJ-124 \\
\hline $\mathbf{1 2 .}$ & VARUNA & 28. & GEETA \\
\hline $\mathbf{1 3 .}$ & MAYA & 29. & BIO-902 \\
\hline $\mathbf{1 4}$ & ROHINI & 30. & RH-819 \\
\hline $\mathbf{1 5}$ & LAXMI & 31. & JMM-927 \\
\hline $\mathbf{1 6 .}$ & RCC-4 & & \\
\hline
\end{tabular}


Appendix.2 List of accession lines used in study

\begin{tabular}{|c|c|}
\hline S.No. & Accession Lines \\
\hline $\mathbf{1 .}$ & IC- -26513 \\
\hline $\mathbf{2 .}$ & IC- 113037 \\
\hline $\mathbf{3 .}$ & IC -264986 \\
\hline $\mathbf{4 .}$ & IC -571648 \\
\hline $\mathbf{5 .}$ & IC- -4052358 \\
\hline
\end{tabular}

Appendix.3 List of 15 SSR markers used in present study

\begin{tabular}{|c|c|c|}
\hline Primer & Forward (5'-3') & Reverse (5'-3') \\
\hline Ni1-A04 & TCСТССТАСТTTGATACT & ACGTCAAATACTTCACTG \\
\hline Ni2- A07 & GGAACCCAACAACAAGTGAG & AGAGCTTGAGACACATAACACC \\
\hline Ni2- C06 & CACTGGGATACAAGCCCTTC & ACAATTTGAAGTACAAACTCTCTC \\
\hline Ni2- C12 & ACATTCTTGGATCTTGATTCG & AAAGGTCAAGTCCTTCCTTCG \\
\hline Ni2- H06 & CATCAGATCCGACGAAATCC & TCCTTTGGACTGTGAAAAACG \\
\hline Ni3- H07 & GCTGTGATTTTAGTGCACCG & AGCCGTTGATGGAATTTTTG \\
\hline Ni4- A03 & ACACAGAAACATCAAACATACC & GGACCGGTTTTATTTGTTCG \\
\hline Ni4- E08 & GATTTTGAGGAAGCGGAGG & CAAAGCACTGAGAGAGAGAGAGAG \\
\hline Ra2-A01 & TTCAAAGGATAAGGGCATCG & TCTTCTTCTTTTGTTGTCTTCCG \\
\hline Ra2-A02 & AACCTCCGACGTGTGTGTG & TCATCACCACCATCACCATC \\
\hline Ra2-A11 & GACCTATTTTAATATGCTGTTTTACG & ACCTCACCGGAGAGAAATCC \\
\hline Ra2-C11 & CGCCTATTTCACACACACAC & GTGTTACACGTCACAACGC \\
\hline Ra2- E12 & TGTCAGTGTGTCCACTTCGC & AAGAGAAACCCAATAAAGTAGAACC \\
\hline Ni2-C01 & GAGTATGAGATGGGAATCCG & GACTGAGCAGCTTGGAGACC \\
\hline Ni2-D07 & ACCAAAGCTGATCTCCAACC & АCTCTTCGAATTCTTTTCC \\
\hline
\end{tabular}

Fig.1 $0.8 \%$ Agarose gel to check DNA integrity.1 to 36 is genotype number loaded on gel as per sequence mentioned in table

$\begin{array}{lllllllllllll}1 & 2 & 3 & 4 & 5 & 6 & 7 & 8 & 9 & 10 & 11 & 12 & 13\end{array}$

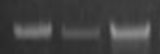

$\begin{array}{lllllllllllll}14 & 15 & 16 & 17 & 18 & 19 & 20 & 21 & 22 & 23 & 24 & 25 & 26\end{array}$

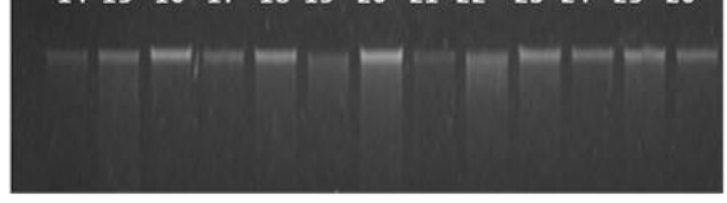

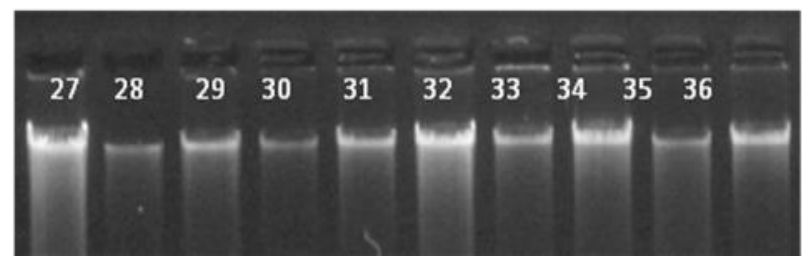


Fig.2 Ethidium bromide stained DNA amplification profile 36 genotypes of Indian mustard using microsatellite marker (Ni3- H07).Lanes 1 to $36=$ genotypes
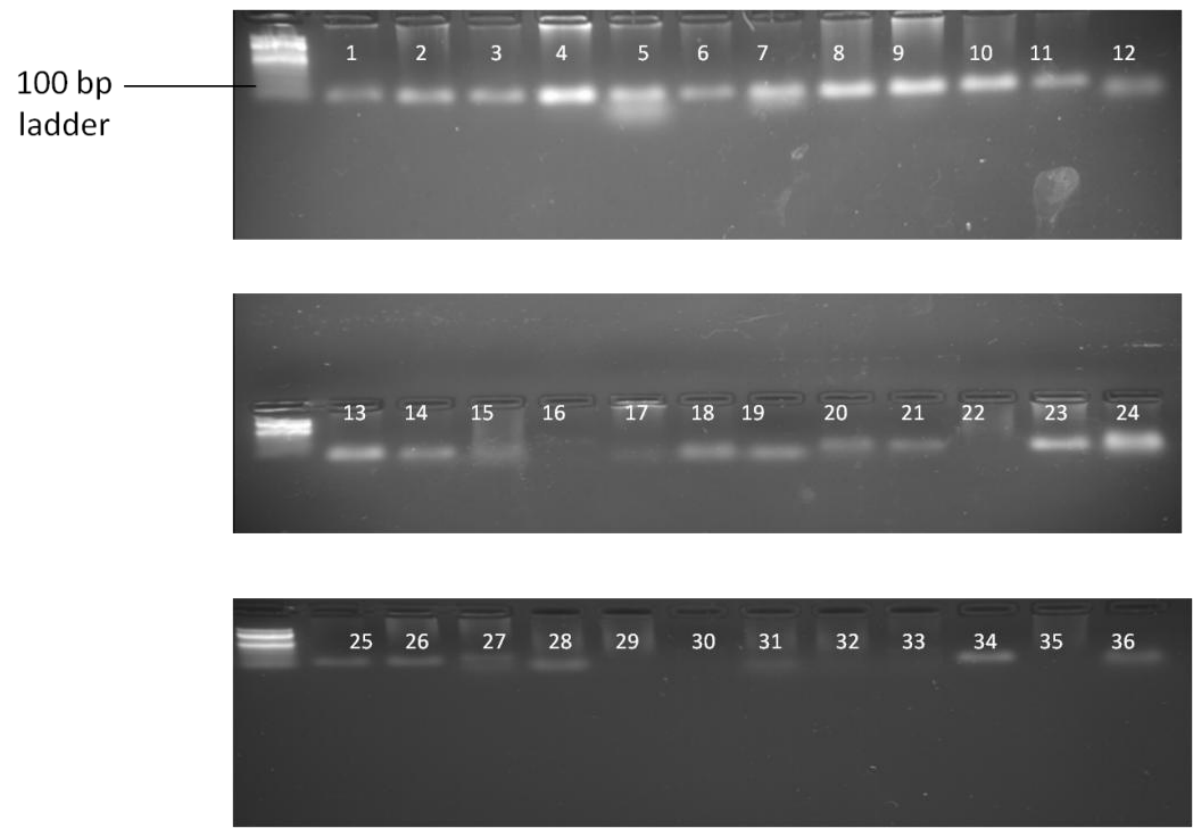

Fig.3 Dendrogram showing Jaccard's dissimilarity produced using UPGMA cluster analysis demonstrating association among 36 genotypes of Indian mustard

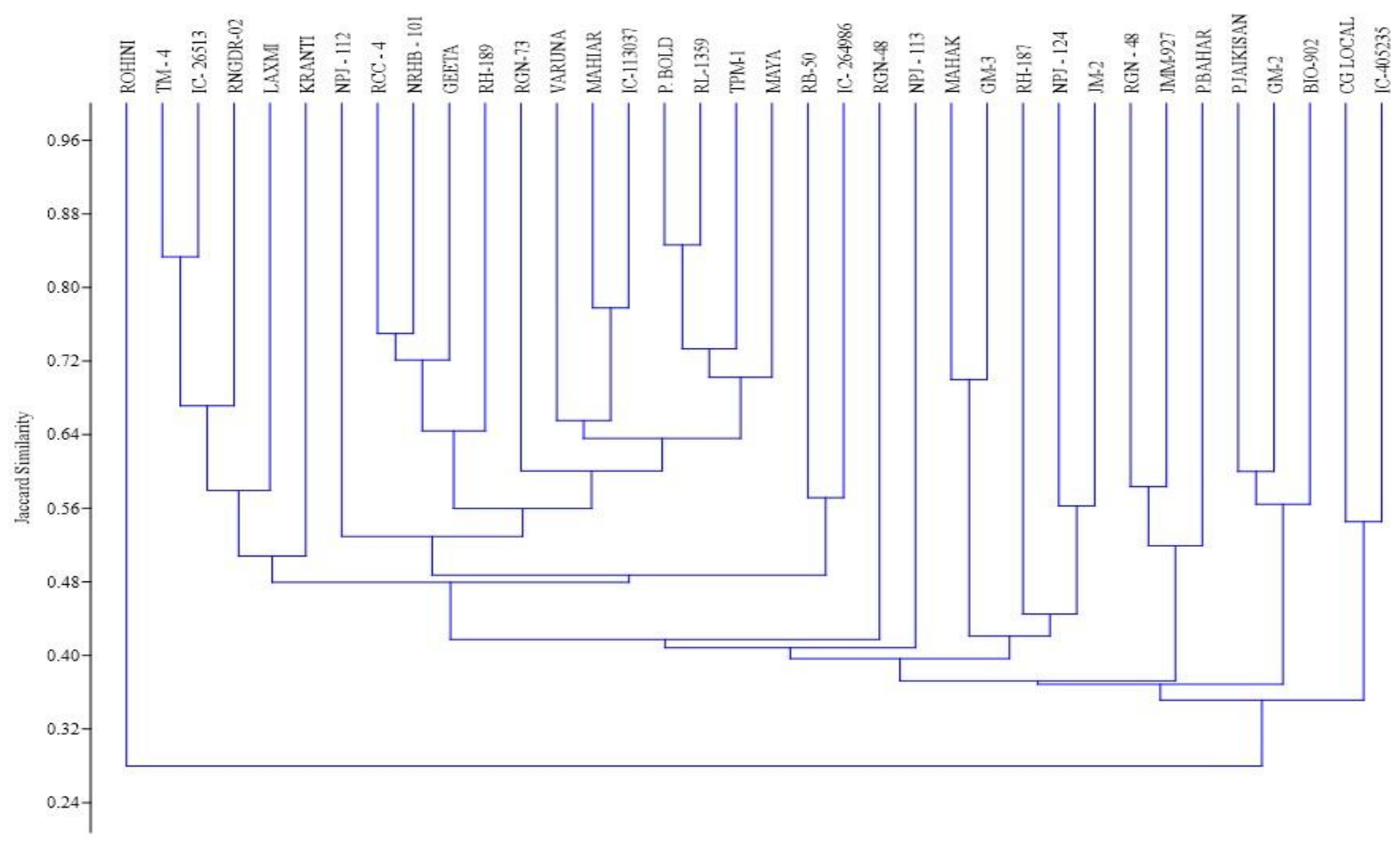


The present study also found that Pusa Mahak is a glossy mutant of P.Bold, developed from IARI, falls in two different clusters as expected. Further, the genotypes GM 3 and JM 2 have Varuna as their immediate or distant ancestor and are present in same sub sub groups.

In this study we found that IARI bred variety Pusa Jaikisan, a somaclone variant of Varuna developed through tissue culture, falls in the cluster away from its parent. A similar result regarding effectiveness of SSR markers in monitoring genetic diversity for yield component traits as well as quality traits have also been reported by Charters et al., (1996) and Plieske and Struss (2001) respectively.

Similar types of studies using SSR markers have also been done in B. napus (Batley et al., 2003; Hopkins et al., 2006). Vinu et al., (2012) also used 143 SSR primers against 44 genotypes of $B$. juncea for assessment of genetic diversity. In addition to microsatellite markers, other marker systems were also used by various researchers for genetic diversity studies in Brassica species. Malode et al., (2010) also analyzed 20 genotypes of Brassica spp. including exotic, Indian and mutants using RAPD primers and they were grouped into four clusters.

In conclusion, identification and utilization of genetic diversity is not only very crucial for improvement of crop but also for preserving germplasm resources for future purposes. Identification of any variation present among genotypes can be done on the basis of phenotypic, physiological and genetic parameters. However, screening of plants based on phenotypic and physiological characters proofs to be time consuming, labour intensive, biased and can't handle large population at a time.

On the other hand DNA markers overcome all the above said drawbacks and are free from environmental fluctuations. They act as a stronger tool in distinguishing between $B$. juncea genotypes. Information on genetic distances obtained from these microsatellite markers can provide a wider opportunity to create selectable and suitable genetic variation using genotypes which are genetically wide apart.

\section{References}

Batley, A.J., Vecchies, A.A., Mogg, B.R., Bond, B.J., Cogan, N.A., Hopkins, C.A., Gororo, N.C., Marcroft, C.S., Forster, A.J., Spangenberg, A.G. and Edwards, A.D. 2003. A study of genetic diversity among Brassica napus and Brassica juncea germplasm collections using Simple Sequence Repeat (SSR) Molecular Markers. 13th Australian Research Assembly on Brassicas Conference Proceedings. Pp. 86-88.

Charters, Y.M., Robertson, A., Wilkinson, M.J. and Ramsay, G. 1996. PCR analysis of oilseed rape cultivars (Brassica napus L. Oleifera) using 5anchored Simple Sequence Repeat (SSR) primers. Theory of Applied Genetics. 92: 442-447.

Doyle, J. J. and Doyle, J. L. 1990. Isolation of Plant DNA from fresh tissue. Focus, 12, 13-15.

Hopkins, C., Mogg, R., Gororo, N., Salisbury, P., Burton, W., Love, C., Spangenberg, G., Edwards, D. and Batley, J. 2006. An assessment of genetic diversity within and between Brassica napus and Brassica juncea lines. Acta Horticulturae. 706: 115-119.

https://www.usda.gov

Hu, S., Yu, C., Zhao, H., Sun, G., Zhao, S., Vyvadilova, M. and Kucera, V. 2007. Genetic diversity of Brassica napus L. Germplasm from China and Europe assessed by some agronomically important characters. Euphytica. 154, 916. 
Kumar, P.R. 2015. Package of practices and contingency plan for enhancing production of rapeseed-mustard in India. A publication of the National Research Centre on Rapeseed-Mustard, Sewar, Bharatpur, Pp 1-39.

Malode, S.N., Shingnapure, S.M., Waghmare, V.N. and Sutar, S. 2010. Genetic diversity analysis of some exotic, Indian and mutant Brassica sp. Through RAPD markers. African Journal of Biotechnology. 9(26): 3981-3987.

Mishra, M.K., Suresh, N., Bhat, A.M., Suryaprakash, N., Kumar, S.S., Kumar, A. and Jayarama. 2011. Genetic molecular analysis of Coffea arabica hybrids using SRAP markers. Revista de Biología Tropical. 59, 607-617.

Mohammadi, S. A. and Prasanna, B. M. 2003. Analysis of genetic diversity in crop plants salient statistical tools and considerations. Review and Interpretation. Crop Science. 43, 12351248.

Plieske, J. and Struss, D. 2001. Microsatellite markers for genome analysis in Brassica. I. Development in Brassica napus and abundance in Brassicaceae species. Theory of Applied Genetics. 102: 689-694.

Vinu, V., Singh, N., Vasudev, S., Yadava, D.K.,Kumar, S., Naresh, S., Bhat, S.R. and Prabhu, K.V. 2013. Assessment of genetic diversity in Brassica juncea genotypes using phenotypic differences and SSR markers. Revista de Biología Tropical. 61(4):1919-34.

www.fao.org/faostat/en/

\section{How to cite this article:}

Nupur Saini and Archana N. Rai. 2020. Molecular Analysis of Diversity Presents in Brassica juncea genotypes with the Help of SSR Markers. Int.J.Curr.Microbiol.App.Sci. 9(07): 29872993. doi: https://doi.org/10.20546/ijcmas.2020.907.352 